\section{NEUROPEPTIDES}

\section{Understanding our fears}

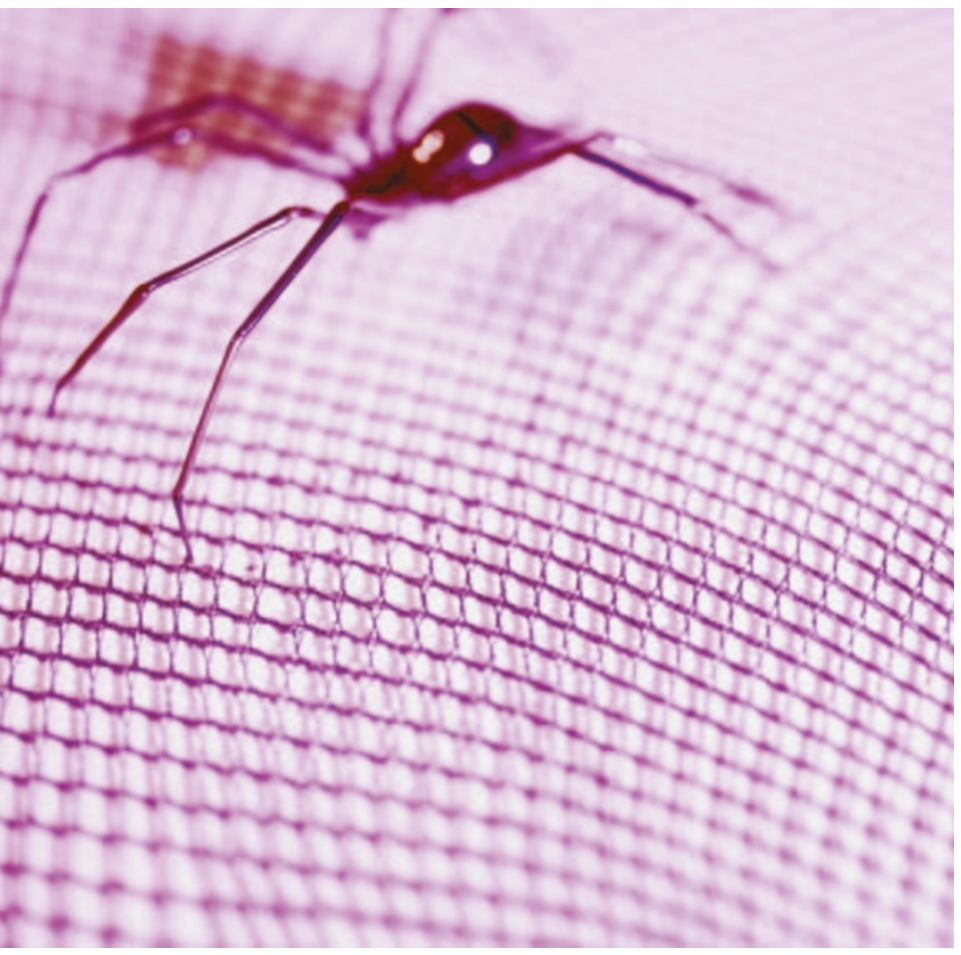

It has long been recognized that the amygdala is involved in the expression of fear in mammals, and that vasopressin and oxytocin can modulate this response. Huber and colleagues have now shown, for the first time, that two distinct neuronal populations in the amygdala are excited by either vasopressin or oxytocin, and that their interactions might represent a neurophysiological mechanism for regulation of the fear response.

The central amygdala (CeA) triggers autonomic fear expression by signalling to the brainstem and hypothalamus, and is known to express various neuropeptide receptors, including those for vasopressin and oxytocin. Vasopressin enhances aggression, anxiety and stress levels, and the consolidation of fear memory. Conversely, oxytocin decreases stress and anxiety, and facilitates maternal care and other social interactions. Both neuropeptides increase neuronal activity in the CeA, but, until now, their mechanism of action was unclear.

The authors first determined the positions of vasopressin and oxytocin receptors within previously defined regions of the CeA - vasopressin receptors in the medial part and oxytocin receptors in the lateral and capsular areas - using autoradiography of rat brain sections. Next, by applying different oxytocin and vasopressin receptor agonists, they discovered two types of responsive neuron: those excited by oxytocin-receptor activation, and those inhibited by oxytocinreceptor activation but excited by a specific vasopressin-receptor type, V1a.

Using sharp-electrode intracellular recordings, the authors determined the precise locations of these neurons in the CeA, and found that vasopressin-excited cells were restricted to the medial CeA whereas oxytocin-excited cells were found in the lateral CeA. This correlated with the autoradiographic map of the receptors. The inhibitory effects of oxytocin on vasopressinexcited cells were caused by the enhanced excitability of neurons in the lateral $\mathrm{CeA}$ areas, which led to an increase in GABA ( $\gamma$-aminobutyric acid) release in the medial region of the CeA.

\title{
Obesity's sleepy link
}

Recent epidemiological studies have noted a link between obesity and insufficient sleep, but the underlying mechanisms are not clear. Now, two reports provide genetic and physiological evidence of how the regulation of circadian rhythm, sleep and metabolism might be tightly coordinated.

Turek and colleagues investigated whether disruption of the circadian system could affect an animal's eating and sleeping behaviours. The circadian system, which controls the timing of almost every aspect of physiology, relies on the so-called 'clock' genes such as clock $(\mathrm{Clk})$, period (Per) and timeless (Tim). The authors found that mice that lacked a functional Clk gene - the primary driver of the circadian machinery - slept less and ate more than their wild-type counterparts. Such mice are heavier and show a significant increase in body fat compared with control animals: $35 \%$ when fed a normal diet and $75 \%$ when fed a high-fat diet. In addition, they show various tissue and biochemical abnormalities that are hallmarks of metabolic disorders, such as high levels of blood glucose and cholesterol, and low levels of insulin. These phenotypes might be related to a decrease in the production of the appetite-regulating hypothalamic hormones leptin and ghrelin in the mutant mice.

In the second study, Hovath and Diano showed that the hypocretin (also called orexin) neurons in the lateral hypothalamus might be a crucial integrator of sleep and metabolism regulation. These neurons secrete hypocretin - a key regulator of both feeding and arousal - and project to many regions of the brain, including the hypothalamus, cerebral cortex, brain stem and spinal cord. Overnight food deprivation promotes the formation of excitatory synapses onto hypocretin neurons and leads to an increase in miniature excitatory postsynaptic currents in these neurons. These effects can be blocked by leptin administration during fasting and are reversed when feeding is subsequently resumed. The results point towards the intriguing possibility that obesity-associated metabolic defects, such as reduced production of leptin, and leptin dysfunction, could render hypocretin neurons more excitable and result in an increased level of arousal and insomnia.

Therefore, genes, hormones and the ability of neurons to undergo synaptic changes can all affect both sleep and metabolism. Further research on the relationships between the two processes and their molecular underpinnings should provide guidelines for the development of new therapeutic approaches to treat obesity and sleep disorders.

Jane Qiu

(2) References and links

ORIGINAL RESEARCH PAPER Turek, F. W. et al. Obesity and metabolic syndrome in circadian Clock mutant mice. Science 21 April 2005 (doi:10.1126/science.1108750) | Horvath, T. L. \& Gao, X. B. Input organization and plasticity of hypocretin neurons: possible clues to obesity's association with insomnia. Cell Metab. 1, 279-285 (2005) FURTHER READING Horvath, T. L. \& Diano, S. The floating blueprint of hypothalamic feeding circuits. Nature Rev. Neurosci. 5, 662-667 (2004) 